\title{
Determination of Mobility and Charge Carriers Concentration from Ionic Conductivity in Sodium Germanate Glasses above and below $T_{g}$
}

\author{
Marcio Luis Ferreira Nascimento ${ }^{1,2}$ \\ ${ }^{1}$ PROTEC/PEI-Postgraduate Program in Industrial Engineering, Department of Chemical Engineering, Polytechnic School, \\ Federal University of Bahia, Rua Aristides Novis 2, Federação, 40210-630 Salvador, BA, Brazil \\ ${ }^{2}$ Vitreous Materials Laboratory, Institute of Humanities, Arts, and Sciences, Federal University of Bahia, Rua Barão de Jeremoabo s/n, \\ Idioms Center Pavilion, Ondina University Campus, 40170-115 Salvador, BA, Brazil
}

Correspondence should be addressed to Marcio Luis Ferreira Nascimento; mlfn@ufba.br

Received 31 October 2012; Accepted 28 November 2012

Academic Editors: M. A. Esteso and X. He

Copyright (C) 2013 Marcio Luis Ferreira Nascimento. This is an open access article distributed under the Creative Commons Attribution License, which permits unrestricted use, distribution, and reproduction in any medium, provided the original work is properly cited.

\begin{abstract}
The ionic conductivity and viscous flow data of $x \mathrm{Na}_{2} \mathrm{O} \cdot(1-x) \mathrm{GeO}_{2}, 0.05<x<0.296$, have been collected in a large temperature range, below and above their glass transition temperatures $\left(T_{g}\right)$. A microscopic model is proposed, assuming that the ionic displacement would result from the migration of interstitial positively charged cationic pairs whose concentration is an activated function of temperature. Below $T_{g}$, their migration is also an activated mechanism, but a "free volume" would prevail above this temperature. This discontinuity in the migration mechanism justifies a Dienes-Macedo-Litovitz (DML) relationship to be representative of conductivity data above $T_{g}$ and an Arrhenius law below. According to this model, the enthalpy deduced by the fit of high temperature data using a DML equation would correspond to the charge carrier formation, whose migration enthalpy, below $T_{g}$, could be deduced by the difference between the activation energy measured in the Arrhenius domain and the charge carrier formation enthalpy. To reduce the number of adjustable parameters numerical values were physically justified. We also applied a complete test for conductivity below $T_{g}$, using the so-called weak electrolyte model, splitting activation enthalpy $E_{\sigma}^{A}$ into formation and migration enthalpies and also explaining the variation of pre-exponential term of conductivity with composition.
\end{abstract}

\section{Introduction}

For glass-forming mixtures in the solid or supercooled liquid state, ionic transport due to alkali cations strongly depends on temperature $T$. The variations of the conductivitytemperature product $\sigma T$ in an Arrhenius representation show two distinct behaviors. At the lowest temperatures, that product follows an activated relationship:

$$
\sigma T=A_{\sigma}^{\text {low }} \exp \left(-\frac{E_{\sigma}^{A}}{R T}\right)
$$

where $A_{\sigma}^{\text {low }}$ and $E_{\sigma}^{A}$ are constants; $R$ is the gas constant.
At higher temperatures, experimental data obey another temperature behavior:

$$
\sigma T=A_{\sigma}^{\text {high }} \exp \left(-\frac{E_{\sigma}^{\prime A}}{R T}\right) \exp \left[-\frac{B_{\sigma}}{R\left(T-T_{0}\right)}\right]
$$

where $A_{\sigma}^{\text {high }}, B_{\sigma}$ and $T_{0}$ are constants. This expression will be deduced in more details below.

Such equation suggests an asymptotic decrease of conductivity towards $T_{0}$ and is similar to the empirical VogelFulcher-Tammann-Hesse (VFTH) relationship originally 
established to describe the viscosity-temperature dependence of molten silicates [1-3]:

$$
\eta=A_{\eta}^{\text {high }} \exp \left[\frac{B_{\eta}^{*}}{R\left(T-T_{0}^{*}\right)}\right],
$$

where $T_{0}^{*} \approx T_{0}<T_{g}$ (the glass transition temperature) is an empirical constant at which the viscosity diverges, and $A_{\eta}^{\text {high }}$ has the same meaning of the preexponential constant of the Arrhenius expression:

$$
\eta=A_{\eta}^{\text {low }} \exp \left(\frac{E_{\eta}^{A}}{R T}\right)
$$

where $A_{\eta}^{\text {low }}$ and $E_{\eta}^{A}$ are constants. The regime of viscosity below $T_{g}$ follows (4) and is called isostructural (i.e., the viscosity of the glassy state where the structure is frozen). Unfortunately (4) was not used because there is no data in such temperature range.

Some authors have correlated $T_{0}$ with the Kauzmann temperature. The $B_{\sigma}$ and $B_{\eta}^{*}$ parameters are erroneously denominated "activation energies." In fact, the activation enthalpy for viscous flow in VFTH depends on $B_{\eta}^{*}$ and $T_{0}$ in the form $B_{\eta}^{*} T /\left(T-T_{0}^{*}\right)$, considering a fixed temperature $T$. As described below, both $B$ parameters are related to "free volumes."

In the high temperature domain a hybrid viscosity form associating (3) and (4) has been found: Macedo and Litovitz [4] determined the average activation energy for viscous flow from the probability of forming a hole. Their ideas resulted in an advantageous equation, that combines both the Arrhenius and VFTH equations with the free volume model $[5,6]$. Some years before, and following analogous considerations, Dienes [7] obtained a similar expression, but using explicitly the equation below, that includes the VFTH term:

$$
\eta=A_{\eta}^{\text {high }} \exp \left(\frac{E_{\eta}^{A}}{R T}\right) \exp \left[\frac{B_{\eta}}{R\left(T-T_{0}\right)}\right]
$$

where $B_{\eta}$ is another constant.

In this work we attribute (5) to Dienes, Macedo and Litovitz (DML). The temperature at which the transport properties change from the Arrhenius law to one governed by (2) or (3) is near $T_{g}$. At this temperature, transport and mechanical properties change from solid-like to liquid-like.

Both viscosity and conductivity data in large temperature ranges are not commonly available since different experimental setups are used below and above $T_{g}$. Nevertheless some results have been published, considering the same batches [8], something rare in the literature. The ionic conductivity and viscous flow behavior for sodium germanate glasses and supercooled liquids at wide temperature range (from below $T_{\mathrm{g}}$ to over $T_{m}$ ) are illustrated in Figures 1 and 2, respectively. Lines correspond to adjustable models explained below.

The purpose of this paper is to verify the validity of two microscopic models, trying to find a connection between conduction and viscous flow processes. These models could

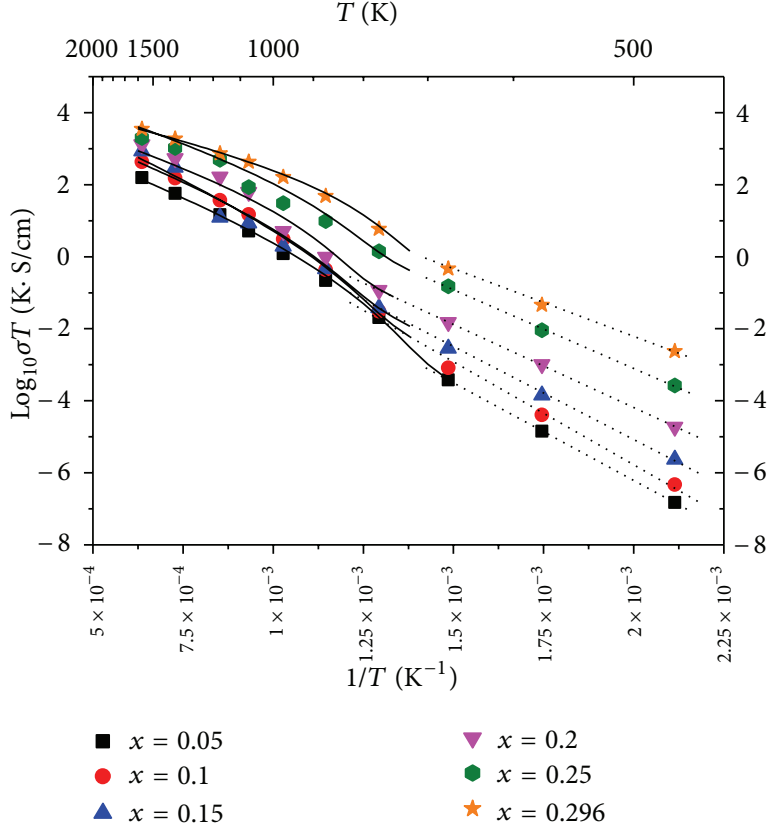

FIGURE 1: Variation of the $\sigma T$ product as a function of temperature in the Arrhenius coordinates for sodium germanate glass system. Full and dashed lines represent the extrapolations of the fits to (1) and (2) for several conductivity data [8].

ascribe a physical meaning to the different ionic conductivity parameters $A_{\sigma}^{\text {high }}, E_{\sigma}^{\prime A}, B_{\sigma}$ and $T_{0}$ in (2) with viscosity parameters $A_{\eta}^{\text {high }}, E_{\eta}^{A}, B_{\eta}$ and $T_{0}$ from (5)-considering the same $T_{0}$ in both cases. We also verified the validity of such obtained parameters from conductivity models above and below $T_{g}$. Careful attention was done to predictable $B_{\eta}$ and $B_{\sigma}$ values. From such conductivity values is possible to assess the activation enthalpy formation, that will be considered on application of the weak electrolyte theory for conductivity data below $T_{g}$, as presented below. We also will take care of the conductivity dependence with composition, obtaining the resulting charge carrier concentration as well as mobilities for each glass system.

\section{Experimental Procedure}

Samples were melted in platinum crucible. In fact, it is easier to measure conductivity data below $T_{g}$ due to different experimental setups: the design of conductivity cells is different below and above the glass transition temperature. Below $T_{g}$, glass samples are generally discs of 1 to $3 \mathrm{~cm}$ in diameter and 1 to $5 \mathrm{~mm}$ thickness. Graphite electrodes were deposited on the two faces in this case study. Electrical measurements were made by d.c. measurements by means of a.c. bridge method using alumina cell with wire electrodes. For conductivity values lower than $10^{-3} \mathrm{~S} / \mathrm{cm}$ and an applied voltage below $200 \mathrm{mV}$, the electrode polarization is low, and no significant differences can be 
TABLE 1: Numerical resulting values from the fits of conductivity to (9) at low temperature, to (23) for high temperature, and viscosity data to (5), considering $A_{\eta}=10^{-5} \mathrm{~Pa} \cdot \mathrm{s}[8]$. Please note that we used the same $T_{0}$ in conductivity as well as viscous flow processes above $T_{g}$.

(a) $T<T_{g}$

\begin{tabular}{lcccc}
\hline$x \mathrm{Na}_{2} \mathrm{O}$ & $A_{\sigma}^{\text {low }}(\mathrm{K} / \Omega \cdot \mathrm{cm})$ & $\Delta H_{f}(\mathrm{eV})$ & $\Delta H_{m}(\mathrm{eV})$ & $E_{\sigma}^{A}(\mathrm{eV})$ \\
\hline 0.05 & $10^{4.616 \pm 0.049}$ & $1.7850 \pm 0.0024$ & $0.1817 \pm 0.0006$ & $1.0742 \pm 0.0055$ \\
0.10 & $10^{5.66 \pm 0.69}$ & $1.9023 \pm 0.0015$ & $0.1828 \pm 0.0012$ & $1.134 \pm 0.081$ \\
0.15 & $10^{5.08 \pm 0.23}$ & $1.6597 \pm 0.0011$ & $0.1791 \pm 0.0041$ & $1.009 \pm 0.027$ \\
0.20 & $10^{5.044 \pm 0.046}$ & $1.4778 \pm 0.0024$ & $0.1771 \pm 0.0008$ & $0.9160 \pm 0.0055$ \\
0.25 & $10^{5.63 \pm 0.27}$ & $1.3796 \pm 0.0038$ & $0.1767 \pm 0.0031$ & $0.866 \pm 0.030$ \\
0.296 & $10^{5.05 \pm 0.21}$ & $1.0955 \pm 0.0097$ & $0.1742 \pm 0.0023$ & $0.722 \pm 0.023$ \\
\hline
\end{tabular}

(b) $T>T_{g}$

\begin{tabular}{|c|c|c|c|c|c|c|c|}
\hline$x \mathrm{Na}_{2} \mathrm{O}$ & $A_{\sigma}^{\text {high }}(\mathrm{K} / \Omega \cdot \mathrm{cm})$ & $E_{\sigma}^{A}=\Delta H_{f} / 2(\mathrm{eV})$ & $B_{\sigma}(\mathrm{eV})$ & $E_{\eta}^{A}(\mathrm{eV})$ & $B_{\eta}(\mathrm{eV})$ & $T_{0}(\mathrm{~K})$ & $T_{g}(\mathrm{~K})$ \\
\hline 0.05 & $10^{4.616 \pm 0.049}$ & $0.8925 \pm 0.0012$ & $0.0180 \pm 0.0097$ & $1.189 \pm 0.049$ & $0.231 \pm 0.012$ & $580 \pm 10$ & 729 \\
\hline 0.10 & $10^{5.66 \pm 0.69}$ & $0.9512 \pm 0.0008$ & $0.0139 \pm 0.0040$ & $1.082 \pm 0.091$ & $0.190 \pm 0.024$ & $669 \pm 21$ & 783 \\
\hline 0.15 & $10^{5.08 \pm 0.23}$ & $0.8299 \pm 0.0005$ & $0.0181 \pm 0.0098$ & $1.081 \pm 0.057$ & $0.177 \pm 0.017$ & $706 \pm 4$ & 791 \\
\hline 0.20 & $10^{5.044 \pm 0.046}$ & $0.7389 \pm 0.0012$ & $0.0129 \pm 0.0097$ & $0.977 \pm 0.011$ & $0.209 \pm 0.014$ & $693 \pm 5$ & 793 \\
\hline 0.25 & $10^{5.63 \pm 0.27}$ & $0.6898 \pm 0.0016$ & $0.0299 \pm 0.0034$ & $0.889 \pm 0.006$ & $0.2655 \pm 0.0024$ & $624 \pm 19$ & 755 \\
\hline 0.296 & $10^{5.05 \pm 0.21}$ & $0.5478 \pm 0.0048$ & $0.0176 \pm 0.0057$ & $0.712 \pm 0.091$ & $0.2887 \pm 0.0061$ & $599 \pm 6$ & 716 \\
\hline
\end{tabular}

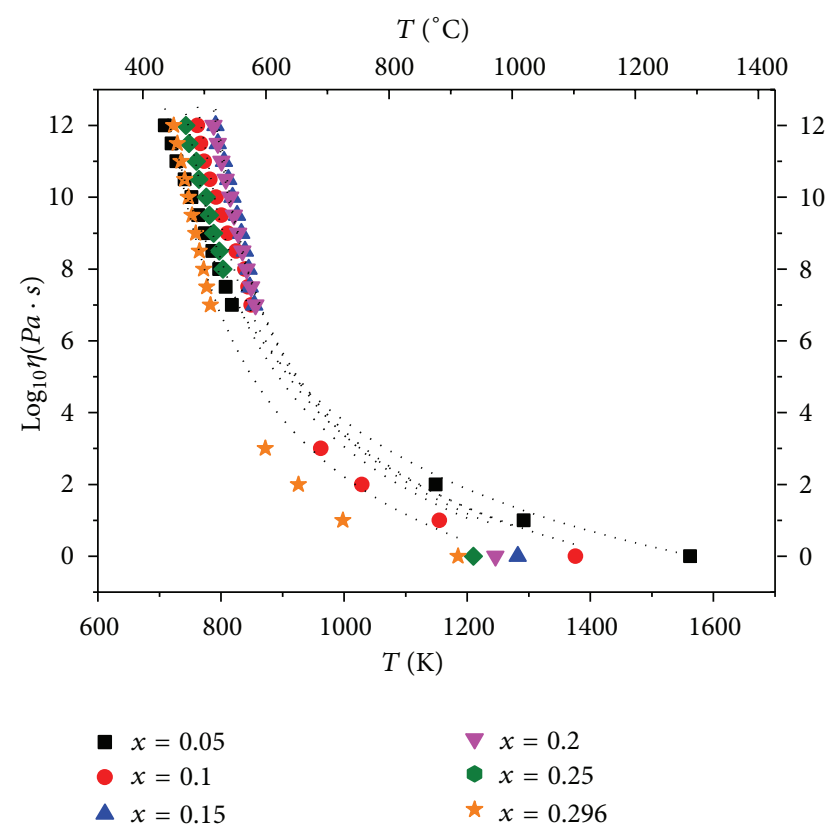

FIGURE 2: Comparison test of experimental viscosity data on binary sodium germanate glass system using the Dienes-Macedo-Litovitz (5) viscosity equation [8].

noticed between the two techniques. At higher temperatures, over $T_{q}$, they are contained in platinum crucibles and two platinum electrodes immersed in the melt. In general the cell is previously calibrated at room temperature with a $\mathrm{KCl}$ solution. To avoid electrode polarization the melt resistance is determined in a frequency range in which the resistance is not frequency dependant, generally around $10 \mathrm{kHz}$.
For obvious technological reasons, the viscosity of a glass or glass-forming melt is an important characteristic justifying the large number of available experimental data. The common viscosity unit is the Poise but the SI unit of viscosity is the $\mathrm{Pa} \cdot \mathrm{s}$ with the correspondence $1 \mathrm{~Pa} \cdot \mathrm{s}=10$ Poise. From an experimental point of view, viscosities are currently determined between $\approx 10^{14}$ and $\approx 10^{-2} \mathrm{~Pa} \cdot \mathrm{s}$. Over a value of $10^{12} \mathrm{~Pa} \cdot \mathrm{s}$ the melt has a solid-like behavior and is called a glass, and, below this value, designed as a liquid or supercooled liquid. Few methods are most frequently used to measure viscosity at low or high viscosity. At low viscosity, up to $10^{4} \mathrm{~Pa} \cdot \mathrm{s}$, the rotating cylinder, counterbalanced method, or crucible techniques appear to be the most reliable. Higher viscosities $\left(>10^{7} \mathrm{~Pa} \cdot \mathrm{s}\right)$ are measured by the rate at which a glass rod elongates under a constant stress or even by penetration/beam-bending methods. The viscosity was measured in this sodium germanate system by counterbalanced method with Pt body and crucible for $\eta \leq 10^{3} \mathrm{~Pa} \cdot \mathrm{s}$ and by penetration method for higher values.

$T_{g}$ was obtained from dilatometric measurements with error $\pm 5 \mathrm{~K}$. More details can be found elsewhere [8]. All adjustments were performed using a Levenberg-Marquardt nonlinear fitting using the Origin software. Table 1 gives the corresponding experimental results, according to theoretical explanation given in this work.

\section{Ionic Transport below the Glass Transition Temperature $\left(T_{g}\right)$}

Below $T_{g}$, the usual approach developed for ionic crystals prevails: charge carrier formation may be described by partial dissociation of ionic pair equivalent to the formation of a Frenkel defect, and their migration in a glass is caused by an indirect interstitial mechanism [9], as briefly explained below. 
The two processes are activated and justify an Arrhenius law for conductivity dependence with $T$ (1).

In this temperature range, and for all ionic conductive glasses, the representation of experimental data of the conductivity-temperature product, $\sigma T$, in the Arrhenius coordinates results in straight lines whose extrapolation towards infinite temperature converges to corresponding values of the preexponential term $A_{\sigma}^{\text {low }}$. Temperature conductivities of sodium germanate glasses under study spread over 12 orders of magnitude only due to the narrow variation of the conductivity activation energy $\left(0.72 \mathrm{eV} \leq E_{A}^{\sigma} \leq 1.13 \mathrm{eV}\right)$, that also depends upon the nature and the concentration of the added alkali cation. Figure 1 shows such conduction results in wide temperature and composition range.

For cationic glasses and regardless of the conduction mechanism, electrical transport can be expressed as the product of three terms, the charge carriers concentration, $n_{+}$, their electrical mobility, $\mu_{+}$, and their charge, $F$ (by means of Faraday constant):

$$
\sigma=F n_{+} \mu_{+}
$$

Since the relative dielectric constant $\varepsilon$ of inorganic silicate glasses is low $(3<\varepsilon<15)$ ionic species are strongly associated. For instance most monovalent cations will be associated with nonbridging oxygen atoms. Such an associated cation should be regarded as in a normal position, hence defining a regular cationic site. Nevertheless, thermal vibrations allow some partial dissociation which leads to the formation of point defects as it is the case in crystalline structures. When a cation leaves its "normal position," it will be in what could be considered an "interstitial position" and its previously occupied site will become a "vacancy." This defect formation in the glass structure is equivalent to the formation of a Frenkel type defect in an ionic crystal. In oxide based glasses for which alkali concentration, $n$, is over $10 \%$ atom (as in this case), "normal" sites are close enough ( $3 \AA$ to $5 \AA$ ) and a leaving cation is now expected to share a neighboring nonbridging oxygen with another cation. This combination of two cations surrounding a nonbridging oxygen can be described as an "interstitial pair defect."

The concentration of these interstitial pairs, $n_{+}$, may be very small compared with the total concentration of alkali cations, $n$. In other words, glasses are weak electrolytes. In that case, the chemical equilibrium between alkali cations in regular sites and interstitial positions leads to the following relationship:

$$
n_{+}=n \exp \left(-\frac{\Delta G_{f}}{2 R T}\right),
$$

where $\Delta G_{f}=\Delta H_{f}-T \Delta S_{f}$ is the free energy associated with the simultaneous formation of an interstitial pair and a cationic "vacancy," $\Delta H_{f}$ is the formation enthalpy, and $\Delta S_{f}$ is the formation entropy. The second step is to describe the defect migration caused by the electric field. Thus, the mobility, $\mu_{+}$, of the charge carrier (the interstitial cation) in the electric field is expressed by the general relationship:

$$
\mu_{+}=\frac{F \lambda^{2} v_{0}}{6 R T} \exp \left(-\frac{\Delta G_{m}}{R T}\right)
$$

In this expression, $\lambda$ is the mean distance between two cationic sites, $\nu_{0}$ the attempt frequency and $\Delta G_{m}=$ $\Delta H_{m}-T \Delta S_{m}$ is characterized by a migration free energy with its corresponding enthalpy $\Delta H_{m}$ and entropy $\Delta S_{m}$.

Using (6), (7), and (8), the cationic conductivity in the low temperature range is given by

$$
\sigma T=n \frac{F^{2} \lambda^{2} v_{0}}{6 R} \exp \left(\frac{\Delta S_{f} / 2+\Delta S_{m}}{R}\right) \exp \left(-\frac{\Delta H_{f} / 2+\Delta H_{m}}{R T}\right) .
$$

This relationship reduces to the experimentally observed the Arrhenius law (1) by identification of the experimental value of $E_{\sigma}^{A}$ to

$$
E_{\sigma}^{A}=\frac{\Delta H_{f}}{2}+\Delta H_{m}
$$

Equation (9) is the usual expression proposed for the intrinsic ionic conductivity-temperature dependence in ionic crystals. The successive steps of a cationic displacement according to this description are illustrated in [10].

Equation (9) interestingly gives the opportunity to calculate a theoretical value for the preexponential term, $A_{\sigma}^{\text {low }}=$ $n\left(F^{2} \lambda^{2} v_{0} / 6 R\right) \exp \left(\left(\Delta S_{f} / 2+\Delta S_{m}\right) / R\right)$. Assuming a homogeneous distribution of the alkali cations through the glass, the jump distance, $\lambda$, is the mean distance between two nonbridging oxygen atoms and is related to the total alkali concentration $\left[\lambda=(1 / n)^{1 / 3}\right]$. For classical oxide glasses, this distance typically varies from $3 \AA$ to $5 \AA$. The attempt frequency, $v_{0}$, may be obtained from the far-infrared absorption which occurs between $500 \mathrm{~cm}^{-1}$ and $100 \mathrm{~cm}^{-1}$ in oxide glasses [11]. Corresponding values of $v_{0}$ are around $10^{13} \mathrm{~Hz}$. Assuming a low value for the entropy terms, $\Delta S_{f}$ and $\Delta S_{m}$, reasonable values of the preexponential term, $A_{\sigma}^{\text {low }}$, lie in the $10^{4}-10^{5}(\mathrm{~K} \cdot \mathrm{S} / \mathrm{cm})$ range in remarkable agreement with most experimental data [12].

From the model above, (10), based on defect crystal theory for ionic transport in solids, the activation enthalpy for conduction $E_{\sigma}^{A}$ is the sum of two enthalpy terms. Contrary to what can be done for ionic conductive materials presenting temperature-dependent intrinsic and extrinsic domains, the migration and formation enthalpies cannot be separated without further experiments and assumptions. In the following paragraphs, we will assume that $\Delta H_{f}$ remains the same at high temperatures and obtain it from viscous flow results considering the same batches.

Fortunately, as far as the author knows, the only theory that can predict the $A_{\sigma}^{\text {low }}$ and $E_{\sigma}^{A}$ composition dependences below $T_{g}$ is the Ravaine-Souquet model $[13,14]$, presented below.

\subsection{Ravaine and Souquet Model (Weak Electrolyte Theory).} The weak electrolyte theory has been extended to glassy electrolytes by Ravaine and Souquet $[13,14]$ to interpret the important variations in conductivity with composition.

In the classical approaches of ionic transport in glasses, all cations, in the present case all $\mathrm{Na}^{+}$, are supposed to 
simultaneously migrate. Since the conductivity variations with composition are of several orders of magnitude larger than the total concentration in $\mathrm{Na}^{+}$cations, these variations in conductivity with composition $(x)$ should be the result of large variations of their mobility with the composition, which cannot be justified from a structural point of view.

Ravaine and Souquet $[13,14]$ have then supposed that the number of instantaneous charge carriers do not correspond to all the $\mathrm{Na}^{+}$cations present in the glass but only to a small fraction of them who temporally escape from their stable cationic site to participate in conduction process. On a long time scale, that is much over the charge carriers life time, all $\mathrm{Na}^{+}$cations participate to ionic transport, but the number of instantaneous charge carriers is far lower than the total number of $\mathrm{Na}^{+}$cations. This hypothesis is similar to ionic transport by defects as previously described for ionic crystals. In the present case, the concentration of instantaneous charge carriers can be identified to interstitial pair concentration $n_{+}$. These interstitial pairs can also be seen as sites of higher energy than the bound lowest energy sites as suggested by Martin and Angell [15] such intermediate sites would temporarily "store" a dissociated cation and make it "available" for ionic conduction.

Thus, Ravaine and Souquet have done the simple hypothesis that the charge carriers mobility $\mu_{+}$could be considered, in a first approximation, as constant with composition in a given glass system and that the large variations in ionic conductivity with composition is the result of the large variations in instantaneous charge carriers concentration with composition. The previous considerations developed remain valid, and the hypothesis of a constant mobility with composition means that all terms in the mobility expression in (9) can be considered as almost constant at constant temperature as a function of $x$.

To estimate, in the $x \mathrm{Na}_{2} \mathrm{O} \cdot(1-x) \mathrm{GeO}_{2}$ system, the number, or at least the variation of instantaneous charge carriers with composition, thermodynamic solutions are applied to this glass-forming system, considered as a "solution" in which the $\mathrm{Na}_{2} \mathrm{O}$ modifier is the solute and $\mathrm{GeO}_{2}$ the "solvent." The charge carriers are obtained by partial dissociation of $\mathrm{Na}_{2} \mathrm{O}$ in this low dielectric solvent like it is the case for the weak electrolyte solutions in electrochemistry. Through this dissociation process, the number of instantaneous charge carriers is an exponential function of the partial free energy $\Delta \bar{G}_{\mathrm{Na}_{2} \mathrm{O}}$ of $\mathrm{Na}_{2} \mathrm{O}$ in the $x \mathrm{Na}_{2} \mathrm{O} \cdot(1-x) \mathrm{GeO}_{2}$ mixture, that is,

$$
n_{+}=n \exp \left(\frac{-\Delta G_{f}^{0}+\Delta \bar{G}_{\mathrm{Na}_{2} \mathrm{O}}}{2 R T}\right),
$$

in which $\Delta G_{f}^{0}$ is the free energy for the formation of a charge carrier in an arbitrary reference state, and $\Delta \bar{G}_{\mathrm{Na}_{2} \mathrm{O}}$ the difference in partial free energy of $\mathrm{Na}_{2} \mathrm{O}$ in this reference state and the studied composition. The factor 2 in the exponent expresses that, similar to ionic crystals, the dissociation process generates two charged species, a charge carrier and its vacant site, both in the same amount.
Finally, the ionic conductivity variations with composition should follow the variations in the sodium partial free energy according to

$$
\log \sigma \approx \log n_{+} \approx \frac{\Delta \bar{G}_{\mathrm{Na}_{2} \mathrm{O}}}{2 R T} .
$$

This correlation has been directly evidenced in different glass-forming systems by simultaneous measurement of the ionic conductivity and alkali partial free energy using potentiometric or calorimetric techniques $[13,16]$. Finally, in this approach, the large variations in ionic conductivity would be the result of large variation in $\Delta \bar{G}_{\mathrm{Na}_{2} \mathrm{O}}$ with composition $(x)$ as long as all other energetic terms may be considered as composition independent.

To interpret these large variations in $\Delta \bar{G}_{\mathrm{Na}_{2} \mathrm{O}}$ with composition, Ravaine and Souquet $[13,14]$ and Pradel et al. [17] have developed a statistical model for binary oxide and sulfide systems similar to those already developed for molten silicates $[18,19]$. Basically, the mixing enthalpy $\Delta H_{\text {mix }}$ is deduced from the enthalpy variation in $\Delta H$ when a bridging oxygen is replaced by two nonbridging oxygens according the equilibrium:

$$
-\underset{\mathrm{I}}{\mathrm{G}}-\mathrm{O}-\underset{\mathrm{I}}{\mathrm{Ge}}-+\mathrm{M}_{2} \mathrm{O} \underset{i i}{\stackrel{i}{\rightleftarrows}}-\underset{\mathrm{I}}{\mathrm{G}}-\mathrm{O}^{-} \mathrm{M}_{\mathrm{M}^{+}}^{\mathrm{O}^{-}}-\underset{\mathrm{I}}{\mathrm{Ge}}-
$$

and the mixing entropy $\left(\Delta S_{\text {mix }}\right)$ estimated from the different possible configurations for the oxygen, that is, ionic $\left(\mathrm{O}^{2-}\right)$, non bridging $\left(-\mathrm{O}^{-}\right)$and bridging $(-\mathrm{O}-)$.

The Gibbs-Duhem equation is then used to calculate the expression of the partial free energy of the alkali oxide $\Delta \bar{G}_{\mathrm{M}_{2} \mathrm{O}}$ as a function of the molar ratio $x(0<x<2 / 3)$ in $\mathrm{M}_{2} \mathrm{O}$ leading to

$$
\begin{aligned}
\Delta \bar{G}_{\mathrm{M}_{2} \mathrm{O}}=R T \log a_{\mathrm{M}_{2} \mathrm{X}}= & \underbrace{\Delta H_{0}+h \frac{2 x}{1-x}}_{\text {enthalpic terms }} \\
& +\underbrace{R T \log \frac{4 x^{2}}{(2-3 x)(2-x)}}_{\text {entropic term }} .
\end{aligned}
$$

The enthalpic term is the sum of two terms, $\Delta H_{0}(<0)$ representing the variations in enthalpy associated to the bond reorganization according to (12)-of the order of some eV [20] - and a corrective term, $h(>0)$, expressing an eventual small decrease in this enthalpy as a function of the number of broken oxygen bridges. In other terms, the electronic reorganization implied in (12) modifies the bond strength of the remaining bridging oxygens as evidenced by X-ray structural analysis [21]. A positive value for $h$ indicates the progressive decrease in the energy balance of the exothermal reaction with the amount of modifier $\mathrm{M}_{2} \mathrm{O}$. This decrease is also evidenced from the reported formation enthalpies of silicates on thermodynamic tables [20].

The dependence of $\Delta \bar{G}_{\mathrm{M}_{2} \mathrm{O}}$ on $x$ and, consequently, the number of charge carriers are due to both the entropic and enthalpic terms. In all cases, the important isothermal 
variations in ionic conductivity with $x$ are well represented by the relationship:

$$
\log \sigma \approx \frac{\Delta \bar{G}_{\mathrm{M}_{2} \mathrm{O}}}{2 R T}=\frac{\Delta H_{0}}{2 R T}+\frac{h}{R T} \frac{x}{1-x}+\frac{1}{2} \log \frac{4 x^{2}}{(2-3 x)(2-x)},
$$

using (14) to express $\Delta \bar{G}_{\mathrm{M}_{2} \mathrm{O}}$ as a function of $x$. An assessment of the enthalpic term $h$ can be done by the research of the best fit with conductivities data as a function of $x$. Such estimation can also be done by fitting variations in activation enthalpy with $x$ since the global activation enthalpy obeys the relation:

$$
E_{\mathrm{RS}}^{A}=E_{0}-h \frac{x}{1-x},
$$

including in the constant term the migration and dissociation enthalpies ( $E_{0}$ is a constant).

Note that in this model, the defect formation and migration energies are not estimated in absolute value; the model only proposes an interpretation of the variations of isothermal conductivity data with the amount of cation modifier dissolved in the $\mathrm{GeO}_{2}$ solvent.

\section{Ionic Transport over the Glass Transition Temperature}

The changes in transport properties above $T_{g}$ involve a cooperative mechanism of the neighboring atoms in addition to the low temperature process, where a new displacement mechanism is observed which may be described by the free volume approach. This mechanism can be understood by a local concentration of free volume distributed throughout the supercooled liquid which leads to the formation of a hole enabling the displacement of the diffusing species. The free volume concept was introduced by Doolittle [5] and further developed by Cohen and Turnbull [6].

At high temperature, that is, above $T_{g}$, in addition to the low temperature process, another displacement mechanism is observed, which is schematized in [10]. Local deformations of the macromolecular chains enable the transfer of the defect to a neighboring position. This deformation requires local fluctuations of the free volume allocated to the chain segments [6]. Let us define $V_{f}^{*}$ as the minimum free volume required in order that the chain movement results in a defect displacement. Hence, the probability, $P_{2}$, that this minimum free volume is reached, is

$$
P_{2}=\exp \left(-\frac{V_{f}^{*}}{\bar{V}_{f}}\right)
$$

where $\bar{V}_{f}$ is the mean free volume allocated to a chain segment. Its temperature dependence can be expressed by $\bar{V}_{f}=V_{0} \alpha\left(T-T_{0}\right)$, where $\alpha$ is the thermal expansion coefficient of the free volume, and $V_{0}$ corresponds to the volume at $T_{0}$.

$P_{2}$ can then be rewritten as

$$
P_{2}=\exp \left[-\frac{V_{f}^{*}}{V_{0} \alpha\left(T-T_{0}\right)}\right]
$$

This expression confirms that this second mechanism only appears above the ideal vitreous transition temperature, $T_{0}$, at which the free volume disappears. Above this temperature, the cationic displacement may occur either by an activated jump $\left(P_{1}=\exp \left(-\Delta H_{m} / R T\right)\right)$ or by an entropic free volume mechanism $\left(P_{2}\right)$. As these mechanisms are exclusive, the total probability of a successful displacement reads

$$
P=P_{1}+P_{2}-P_{1} P_{2}
$$

Under such conditions, the cationic mobility becomes

$$
\mu_{+}=\frac{F \lambda^{2} v_{0}}{6 R T} P
$$

and the cationic conductivity can be rewritten in the following manner:

$$
\begin{aligned}
\sigma T=A_{\sigma}^{\mathrm{high}} \exp \left(-\frac{\Delta H_{f}}{2 R T}\right) & \left\{\exp \left(-\frac{\Delta H_{m}}{R T}\right)\right. \\
& +\exp \left[-\frac{B_{\sigma}^{*}}{R\left(T-T_{0}\right)}\right] \\
& \left.\times\left[1-\exp \left(-\frac{\Delta H_{m}}{R T}\right)\right]\right\} .
\end{aligned}
$$

The variation of this mobility as a function of temperature depends on the relative values of $P_{1}$ and $P_{2}$. According to (18), a free volume migration mechanism is possible as soon as the temperature reaches $T_{0}$. Nevertheless, it is detectable only for higher temperatures because the low temperature activated mechanism prevails below a particular temperature, $T_{g}$, which can be seen as the "electrical vitreous temperature." It is to say that, in the particular temperature range $\left(T_{0}<T<\right.$ $\left.T_{g}\right), P_{2}$ remains very small compared to $P_{1}$, and the mobility expression (20) is reduced to (8). Hence, the conductivity expression is the same as the activated form proposed in (9), keeping its continuity from $T>T_{0}$ up to $T_{m}$ (in the mathematical sense). On the contrary, over $T_{g}, P_{2}$ becomes preponderant and the conductivity expression now reads

$$
\sigma T=A_{\sigma}^{\text {high }} \exp \left(-\frac{\Delta H_{f}}{2 R T}\right) \exp \left[-\frac{V_{f}^{*}}{V_{0} \alpha\left(T-T_{0}\right)}\right]
$$

or

$$
\sigma T=A_{\sigma}^{\text {high }} \exp \left(-\frac{\Delta H_{f}}{2 R T}\right) \exp \left[-\frac{B_{\sigma}}{R\left(T-T_{0}\right)}\right] .
$$

This expression of $\sigma T$ is the product of two exponential terms where the first is representative of an activated mechanism and where the second describes a VFTH behavior. This kind of relationship has already been proposed to improve the viscosity-temperature fits for $\mathrm{B}_{2} \mathrm{O}_{3}$ or alkali silicate above their vitreous transition temperature [4]. We may also note that the preexponential term in this relationship contains the same microscopic characteristic parameters as in (9). It means that the limit of the conductivity-temperature product when the temperature tends to infinity does not depend on 
the nature of the displacement mechanism. Please note that above $T_{g}$ we assume that all activation enthalpy is due to the defect formation and will assume $\Delta H_{f}$ the same as below $T_{g}$. The migration term is mainly due to the "chain segment" motion, that is, due to the formation of holes according to the free volume theory.

\section{Numerical Fits and Discussion}

For all glass compositions studied within the measured temperature range below $T_{g}$, the conductivity was found to obey an Arrhenius behavior, according to (1). Examples are shown in Figure 1, where it is possible to note the increasing in $\sigma T$ when more sodium is added. For conductivity, the initial procedure was to obtain from the Arrhenius fit below $T_{g}$ the activation enthalpy $E_{\sigma}^{A}$. We have reported in Figure 3 the variations of $E_{\sigma}^{A}$ calculated from experimental data using (16) in the range $0.05<x<0.296$ and observed a smooth decrease of $E_{\sigma}^{A}$ between 1.13 to $0.72 \mathrm{eV}$ (see Table 1). On the same graph are superimposed the expected calculated variations in $E_{\mathrm{RS}}^{A}$ deduced from the Ravaine-Souquet model, with $E_{0}=1.187 \pm 0.039 \mathrm{eV}$ and $h=1.04 \pm 0.15 \mathrm{eV}$, giving reasonable results that can be comparable to other systems using the same approach [17]. Please note that this model does not allow to calculate the activation enthalpy in absolute value, but only its variations with composition through the parameter $h$. As far as the author knows, only recently binary silicate systems had activation enthalpy analysis compared with other theoretical models in near the same wide compositional and conduction ranges [22-24].

On Figure 4 are reported the variations of $A_{\sigma}^{\text {low }}$ as a function of $x$ for the compositions investigated. Despite some scattering, one observes a continuous and monotonous increase with $x$, indicating the low influence of enthalpic terms, as expected. This trend was observed and explained in the same way in lithium silicate systems [25]. The full line superimposed represents the expected variations calculated from the weak electrolyte model. For instance, a variation of preexponential term with composition was experimentally observed in binary alkali silicate, borate, germanate, and tellurite systems [26-29], ranging up to two orders of magnitude, with an average value of $10^{4} \mathrm{~K} \cdot \mathrm{S} / \mathrm{cm}$.

Before analyzing the conductivity data above $T_{g}$, we analyzed first the viscous flow behavior and assess reasonable values using $\operatorname{DML}\left(A_{\eta}^{\text {high }}, E_{\eta}^{A}, B_{\eta}, T_{0}\right)$ parameter equation, as shown in Figure 2 and Table 1, using a least square analysis method. As DML and VFTH expressions give the same preexponential $A_{\eta}^{\text {high }}$, we analyzed the viscous flow behavior using it freely, and the average value found was $A_{\eta}^{\text {high }}=$ $10^{-5} \mathrm{~Pa} \cdot \mathrm{s}$, in agreement with experimental analysis done in dozens of silicate and borate systems [30,31]. From this table, it was possible to calculate $E_{\eta}^{A}, B_{\eta}$ and $T_{0}<T_{g}$, resulting in reasonable values, giving one idea of the chemical bond reorganization above $T_{g}$ in this system, because $E_{\eta}^{A}$ ranged between $0.712<x<1.189$. The DML equation uses four parameters and fits better than VFTH to experimental data,

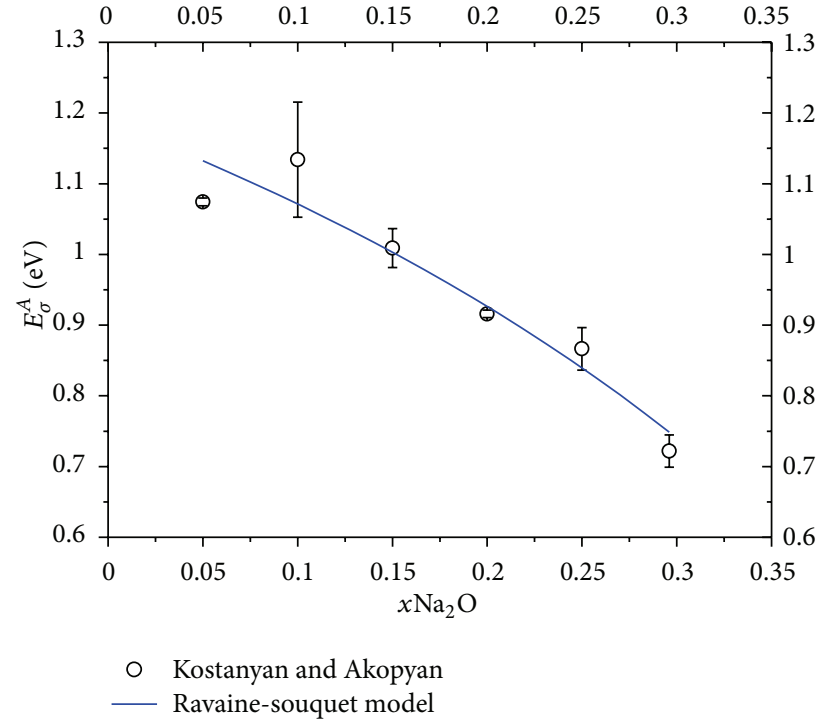

FIGURE 3: Activation enthalpy from experimental data in Figure 1 and (1) as a function of $\mathrm{Na}_{2} \mathrm{O}$ molar ratio $x$, and comparison with calculated values using the Ravaine-Souquet approach (full line).

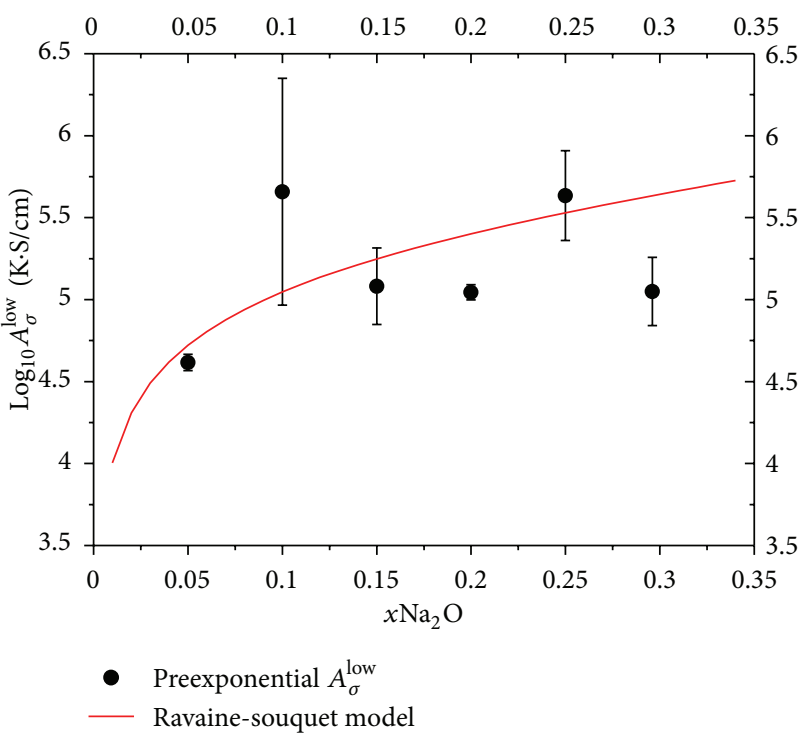

FIgURE 4: Variations of $\log _{10} A_{\sigma}^{\text {low }}$ as a function of $x$ for the compositions investigated. The full line superimposed represents the expected variation calculated from the weak electrolyte model.

but this should be not surprising because the description of experimental results is most accurate by the equations that use the highest number of adjustable parameters.

About conductivity data above $T_{g}$, it was possible to obtain the formation enthalpy term assuming that $E_{\sigma}^{\prime A} \approx \Delta H_{f} / 2$, as shown in Figure 1, thus easily finding the migration term $\Delta H_{m}$. From this procedure we assumed that the defect formation does not depend on the nature of the displacement mechanism, that is, the knowledge of the formation and migration enthalpies was supposed to be the 
same below and above $T_{g}$. From Table 1 it is possible to note that $E_{\sigma}^{A}$ decreased with increasing $x$, physically because less enthalpy is necessary to surpass the kinetic barrier, and near following the same trend observed below $T_{g}$.

We may note that the respective orders of the magnitude of the two enthalpies $\left(\Delta H_{f}\right.$ and $\left.\Delta H_{m}\right)$ are reasonably in agreement with those generally observed in crystalline materials presenting extrinsic and intrinsic conductivity domains which allow, in that case, the separation of the two contributions [32]. It was not possible to obtain the entropy values $\Delta S_{f}$ and $\Delta S_{m}$ by adjusting (9); thus its contribution for preexponential term $\exp \left[\left(\Delta S_{f} / 2+\Delta S_{m}\right) / R\right]$ was assumed inserted in preexponential. Then, the Gibbs free energies $\Delta G_{f}$ and $\Delta G_{m}$ could not be determined from appropriated choice of respective enthalpies and entropies of formation and migration, respectively. Indeed, the preexponential values obtained from the Arrhenius fits was near to $A_{\sigma}^{\text {low }} \approx$ $10^{5} \mathrm{~K} \cdot \mathrm{S} / \mathrm{cm}$, in fact very reasonable results, as explained above.

The coherence between the low and high temperature conductivity parameters was obtained from experimental conductivity data using (23) and considering the same $T_{0}$ from viscosity data (5). We observed that, above $T_{g}, E_{\sigma}^{A}<E_{\eta}^{A}$ and it was possible to obtain easily $B_{\sigma}$ assuming $A_{\sigma}^{\text {high }} \approx$ $A_{\sigma}^{\text {low }}$ fixed, that is, because this term should have the same physical meaning in the full temperature range. All results are summarized in Table 1 . The obtained $B_{\sigma}$ 's values are lower than $B_{\eta}$ considering DML equation, which means that the conduction process needs a lower free volume than the viscous flow. Unfortunately there are no viscosity measurements below $T_{g}$ to verify if the activation enthalpy for conductivity is lower than that for $\eta$.

To be on the safe side we also tested (21), that involves the calculation of the $P_{1}$ and $P_{2}$ temperature dependent probabilities. As the $P_{2} / P_{1}$ ratio shows a sharp increasing above $T_{g}$ in the systems investigated, it confirms the use of the simplified form, (23). For instance, we found that the resulting $B_{\sigma}^{*}$ values from (21) were similar to $B_{\sigma}$, which means that the migration enthalpy is related to the free volume available above $T_{g}$, following similar procedure done by Caillot et al. [33].

We may now emphasize that (21) and (23) contain the usual VFTH "contribution" (3). The multiplying Arrhenius term $\exp \left(E_{\eta}^{A} / R T\right)$ takes into account the formation of defects; in fact this is an advantageous equation, that combines both the Arrhenius and VFTH equations with the free volume model. Such combinations of equations with two different exponential temperature dependencies may reflect different aspects of viscous flow mechanisms, for example, separation of the individual diffusing units from their neighbors followed by a translation over certain distances intro an appropriated vacancy [34].

5.1. An Assessment for Charge Carriers Concentration and Mobility above and below the Glass Transition Temperature. Table 1 allows a comparison between the enthalpies for

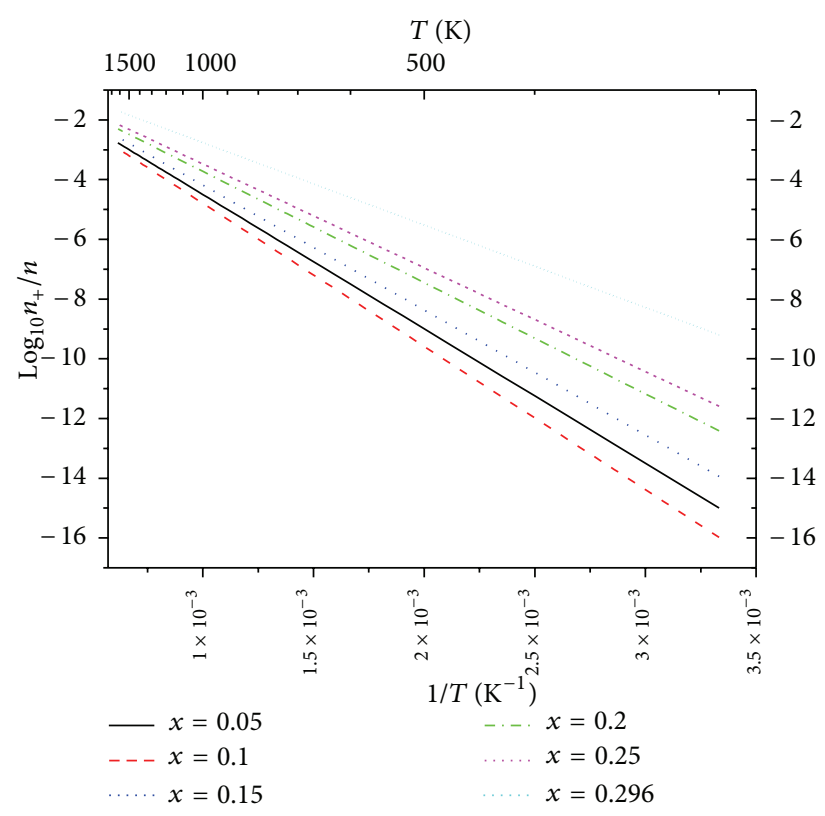

FIGURE 5: The determination of the charge carrier ratio $n_{+} / n$ from the data in Table 1 following (7) in wide temperature and composition ranges.

charge carriers formation and migration deduced from the experimental values for activation enthalpy above and below $T_{g}$. For the investigated compositions, the charge carriers formation require a formation enthalpy of about $1.10-1.90 \mathrm{eV}$, their migration enthalpies, a near fixed value close to $0.18 \mathrm{eV}$.

Using now these experimental values it is thus possible to estimate the relative concentration $n_{+} / n$ of the charge carriers in the glassy state using (7) and their mobility $\mu_{+}$by means of (8). Variations of the relative concentration in charge carriers and their mobility are represented in Figures 5 and 6 . These results are similar to analysis done in alkali disilicates [35]. Note that the combination of both figures gives the previous lines shown in Figure 1.

Mobility values at room temperature are quite similar to each other (near $10^{-4} \mathrm{~cm}^{2} \mathrm{~s}^{-1} \mathrm{~V}^{-1}$ ), due to the same obtained values for migration enthalpy, $\Delta H_{m}$. Such results are similar to the unique mobility measurement of silver done in glass systems, by means of the Hall effect on $x \mathrm{AgI} \cdot(1-x) \mathrm{AgPO}_{3}$ glasses [36]. The experimental value for mobility obtained from $0<x<0.5$ was $(6 \pm 2) \times 10^{-4} \mathrm{~cm}^{2} / \mathrm{V} \cdot \mathrm{s}$ at $25^{\circ} \mathrm{C}$. Thus, our mobility results are in agreement with the hypothesis formulated by the weak electrolyte theory, that is, the independence of the mobility of the ionic carrier with glass composition. Analysis performed in such systems [37] showed similar results and conclusions as done in the present work.

In ionic crystals, the separation of the charge carriers formation enthalpy from the charge carriers migration enthalpy is done by the comparison of the conductivity activation enthalpies in the intrinsic and extrinsic domains [32]. The difference in activation enthalpy in the two domains 


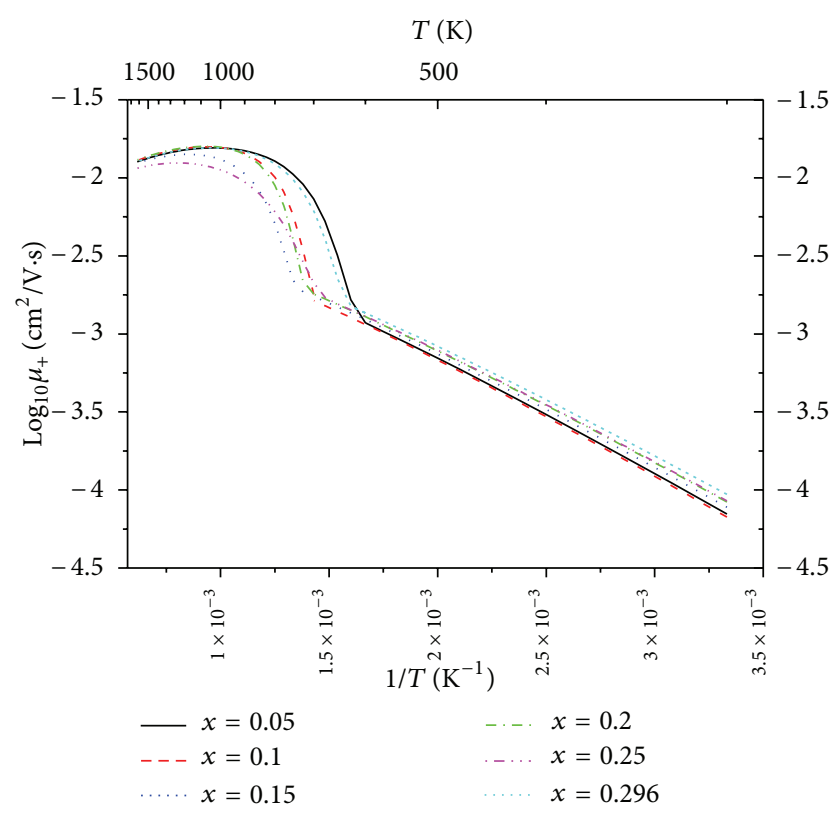

FIGURE 6: Determination of the mobility $\mu_{+}$from data in Table 1 following (8) in wide temperature and composition ranges.

corresponds to the defect formation enthalpy. In other words, for ionic crystals, it is a discontinuity in the origin of the charge carriers which allows to separate the two enthalpic terms. In the present case, it is not a discontinuity in the origin of the charge carriers formation but a discontinuity in the migration mechanism, over and below the glass transition temperature, which allows to separate the two contributions.

A possible error source on our calculations would be on fixing the jumping distance $\lambda=3.5 \AA$ for all systems under study, following (7). Other possible error concerns with few experimental data above $T_{g}[8]$. Finally, the obtained $B_{\sigma}<B_{\eta}$ values are quite acceptable, and it is possible to conclude that the proposal determination route to distinguish activation enthalpies of formation and migration is valid.

\section{Conclusions}

We verified two microscopic models developed to interpret both the ionic conductivity and viscosity variations in large temperature range surrounding the glass transition delimiting the supercooled liquid and vitreous solid domains. Below $T_{g}$, the analyzed model shows that the conductivity obeys an Arrhenius law and that the relevant activation enthalpy $E_{\sigma}^{A}$ is a simple function of the migration and formation enthalpies. Above the ideal glass transition temperature $T_{0}$ a cooperative displacement process, described by the free volume approach, is superposed onto the activated mechanism. Microscopically, this implies that the ionic displacement occurs either because the interstitial pair jumps over an energy barrier separating two adjacent sites or because this energy barrier vanishes consequently to a local rearrangement of surrounding atoms. The latter mechanism results in a conductivity increase appearing for temperatures higher than the transition temperature $T_{g}$. A numerical fit of experimental data to viscosity-temperature model enables the determination of the characteristic parameters of both migration and formation processes, that were used to compare with similar model applied to conductivity data in the same temperature range. Moreover, by means of this identification, it becomes possible to calculate separately the migration and formation enthalpies. All determined preexponential parameters for viscosity and conductivity are consistent with the values expected from our current knowledge of ionic crystals and supercooled liquids.

About the conductivity mechanism below $T_{g}$, the main interest of the Ravaine-Souquet model is to introduce, in addition, the concepts of solution and statistical thermodynamics to quantitatively justify the variations of the concentration in charge carriers with composition. This thermodynamic approach allows to justify the variations of the preexponential term and activation enthalpy with composition. Moreover, the weak electrolyte theory appears to be confirmed by this work, because the mobility of the ionic charge carriers is independent of their concentration, and the determination route to split activation enthalpies seems to work well, giving reasonable results. This work aims to contribute to future discussions to connect the full temperature dependence of the ionic conductivity from below $T_{g}$ to above $T_{g}$.

\section{Acknowledgments}

The author acknowledges the Brazilian agencies FAPESP, Grants 04/10703-0 \& 07/08179-9, and CNPq, contracts 305373/2009-9, 479799/2010-5, 300938/2012-8 and 471546/ 2012-7. The author offers special thanks to Professor Dr. Jean-Louis Souquet, INP-Grenoble, for explaining the weak electrolyte theory during his stay in Brazil and to Professor Dr. Vladimir Mikhailovich Fokin, Vavilov State Optical Institute/Russia, for constant encouragement.

\section{References}

[1] H. Vogel, "The law of the relationship between viscosity of liquids and the temperature," Physikalische Zeitschrift, vol. 22, pp. 645-646, 1921.

[2] G. Tamman and W. Hesse, "Die Abhängigkeit der viscosität von der temperatur bie unterkühlten flüssigkeiten," Zeitschrift für Anorganische und Allgemeine Chemie, vol. 156, pp. 245-257, 1926.

[3] G. S. Fulcher, "Analysis of recent measurements of the viscosity of glasses," Journal of the American Ceramic Society, vol. 8, no. 6, pp. 339-355, 1925.

[4] P. B. Macedo and T. A. Litovitz, "On the relative roles of free volume and activation energy in the viscosity of liquids," Journal of Chemical Physics, vol. 42, p. 245, 1965.

[5] A. K. Doolittle, "Studies in newtonian flow-II. the dependence of the viscosity of liquids on free-space," Journal of Applied Physics, vol. 22, no. 12, pp. 1471-1475, 1951. 
[6] M. H. Cohen and D. Turnbull, "Molecular transport in liquids and glasses," Journal of Chemical Physics, vol. 31, p. 1164, 1959.

[7] G. J. Dienes, "Activation energy for viscous flow and short-range order," Journal of Applied Physics, vol. 24, no. 6, pp. 779-782, 1953.

[8] A. D. Akopyan, Issledovanie Fiziko-Khimicheskikh Svoistv Stekol Sistemy $\mathrm{Na}_{2} \mathrm{O}-\mathrm{RO}-\mathrm{GeO}_{2}$ i Razrabotka na ikh Osnove Novykh Sostavov dlya Vakuumnoi Tekhniki [Ph.D. thesis], Advisor: K. A. Kostanyan, Yerevan State University, 1973.

[9] J. L. Souquet and W. G. Perera, "Thermodynamics applied to ionic transport in glasses," Solid State Ionics, vol. 40-41, no. 2, pp. 595-604, 1990.

[10] J. L. Souquet, M. Duclot, and M. Levy, "Ionic transport mechanisms in oxide based glasses in the supercooled and glassy states," Solid State Ionics, vol. 105, pp. 237-242, 1998.

[11] E. I. Kamitsos, M. A. Karakassides, and G. D. Chryssikos, "Cation-network interactions in binary alkali metal borate glasses. A far-infrared study," Journal of Physical Chemistry, vol. 91, no. 22, pp. 5807-5813, 1987.

[12] G. D. L. K. Jayasinghe, D. Coppo, P. W. S. K. Bandaranayake, and J. L. Souquet, "Electrical properties of $\mathrm{TeO}_{2}$ glasses with $\mathrm{Na}_{2} \mathrm{O}$ as network modifier," Solid State Ionics, vol. 76, no. 3-4, pp. 297 300, 1995.

[13] D. Ravaine and J. L. Souquet, "A thermodynamic approach to ionic conductivity in oxide glasses-part 1 . Correlation of the ionic conductivity with the chemical potential of alkali oxide in oxide glasses," Physics and Chemistry of Glasses, vol. 18, no. 2, pp. 27-31, 1977.

[14] D. Ravaine and J. L. Souquet, "A thermodynamic approach to ionic conductivity in oxide glasses-part 2. A statistical model for the variations of the chemical potential of the constituents in binary alkali oxide glasses," Physics and Chemistry of Glasses, vol. 19, no. 5, pp. 115-120, 1978.

[15] S. W. Martin and C. A. Angell, "Dc and Ac conductivity in wide composition range Li2O.P2O5 glasses," Journal of NonCrystalline Solids, vol. 83, no. 1-2, pp. 185-207, 1986.

[16] J. C. Reggiani, J. P. Malugani, and J. Bernard, "Etude des systèmes vitreaux AgPO3-AgX $(\mathrm{X}=\mathrm{I}, \mathrm{Br}, \mathrm{Cl})$ par calorimétrie de dissolution. Correlation entre l'actvite thermodynamique de AgX et la conductivité ionique du verre," Journal de Chimie Physique, vol. 75, no. 9, pp. 849-854, 1978.

[17] A. Pradel, F. Henn, J. L. Souquet, and M. Ribes, "Use of a thermodynamic model to interpret $\mathrm{Li}+$ ionic conduction in oxide and sulphide binary glasses," Philosophical Magazine B, vol. 60, no. 6, pp. 741-751, 1989.

[18] C. I. B. Fincham and F. D. Richardson, "The behaviour of sulphur in silicate and aluminate melts," Proceedings of the Royal Society of London, vol. 223, pp. 40-62, 1954.

[19] G. W. Toop and C. Samis, "Part II-papers-hydride habit in zirconium and in unstressed and stressed zircaloy-4," Transactions of the Metallurgical Society of AIME, vol. 224, p. 878, 1962.

[20] O. Kubaschewski, F. E. Evans, and C. B. Alcock, Metallurgical Thermochemistry, Pergamon, 1967.

[21] N. Umesaki, N. Iwamoto, M. Tatsumisago, and T. Minami, "A structural study of rapidly quenched glasses in the system $\mathrm{Li}_{2} \mathrm{OSiO}_{2}$," Journal of Non-Crystalline Solids, vol. 106, no. 1-3, pp. 77-80, 1988.

[22] M. L. F. Nascimento, "Test of the Anderson Stuart model and correlation between free volume and the universal conductivity in sodium silicate glasses," Journal of Materials Science, vol. 42, pp. 3841-3850, 2007.
[23] M. L. F. Nascimento and S. Watanabe, "Test of the Anderson Stuart model and correlation between free volume and the universal conductivity in potassium silicate glasses," Materials Chemistry and Physics, vol. 105, pp. 308-314, 2007.

[24] M. L. F. Nascimento, E. do Nascimento, and S. Watanabe, "Test of Anderson-Stuart model and the "Universal" conductivity in Rubidium and cesium silicate glasses," Brazilian Journal of Physics, vol. 35, pp. 626-631, 2005.

[25] M. L. F. Nascimento, A. C. M. Rodrigues, and J. L. Souquet, "Microscopic and thermodynamic interpretations of experimental data on ionic conductivity in lithium silicate glasses," Physics and Chemistry of Glasses, vol. 51, no. 1, pp. 69-77, 2010.

[26] M. L. F. Nascimento and S. Watanabe, "“Universal” curve of ionic conductivities in binary alkali silicate glasses," Journal of Materials Science, vol. 40, no. 18, pp. 5079-5081, 2005.

[27] M. L. F. Nascimento and S. Watanabe, “"Universal” curve of ionic conductivities in binary alkali borate glasses," Journal of Materials Science, vol. 40, no. 16, pp. 4423-4425, 2005.

[28] M. L. F. Nascimento, E. do Nascimento, and S. Watanabe, " "Universal" curve of ionic conductivities in binary alkali germanate glasses," Materials Chemistry and Physics, vol. 96, pp. 55-58, 2006.

[29] M. L. F. Nascimento and S. Watanabe, "Universal curve of ionic conductivities in binary alkali tellurite glasses," Brazilian Journal of Physics, vol. 36, pp. 795-798, 2006.

[30] M. L. F. Nascimento and C. Aparício, "Viscosity of strong and fragile glass-forming liquids investigated by means of principal component analysis," Journal of Physics and Chemistry of Solids, vol. 68, pp. 104-110, 2007.

[31] M. L. F. Nascimento and C. Aparicio, "Data classification with the Vogel-Fulcher-Tammann-Hesse viscosity equation using correspondence analysis," Physica B, vol. 398, no. 1, pp. 71-77, 2007.

[32] F. A. Kröger, The Chemistry of Imperfect Crystals, North Holland, Amsterdam, The Netherlands, 1964.

[33] E. Caillot, M. J. Duclot, J. L. Souquet, M. Levy, F. G. K. Baucke, and R. D. Werner, "Unified model for ionic transport in alkali disilicates below and above the glass transition," Physics and Chemistry of Glasses, vol. 35, no. 1, pp. 22-27, 1994.

[34] I. Gutzow and J. W. P. Schmelzer, The Vitreous State, Springer, 1995.

[35] J. L. Souquet, M. L. F. Nascimento, and A. C. M. Rodrigues, "Charge carrier concentration and mobility in alkali silicates," Journal of Chemical Physics, vol. 132, Article ID 034704, 2010.

[36] V. Clément, D. Ravaine, C. Déportes, and R. Billat, "Measurement of Hall mobilities in AgP03 Agi glasses," Solid State Ionics, vol. 28-30, pp. 1572-1578, 1988.

[37] A. C. M. Rodrigues, M. L. F. Nascimento, C. B. Bragatto, and J. L. Souquet, "Charge carrier mobility and concentration as a function of composition in AgPO3-AgI glasses," Journal of Chemical Physics, vol. 135, Article ID 234504, 2011. 


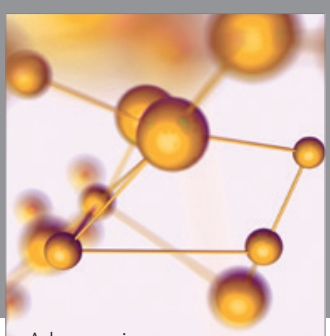

Physical Chemistry
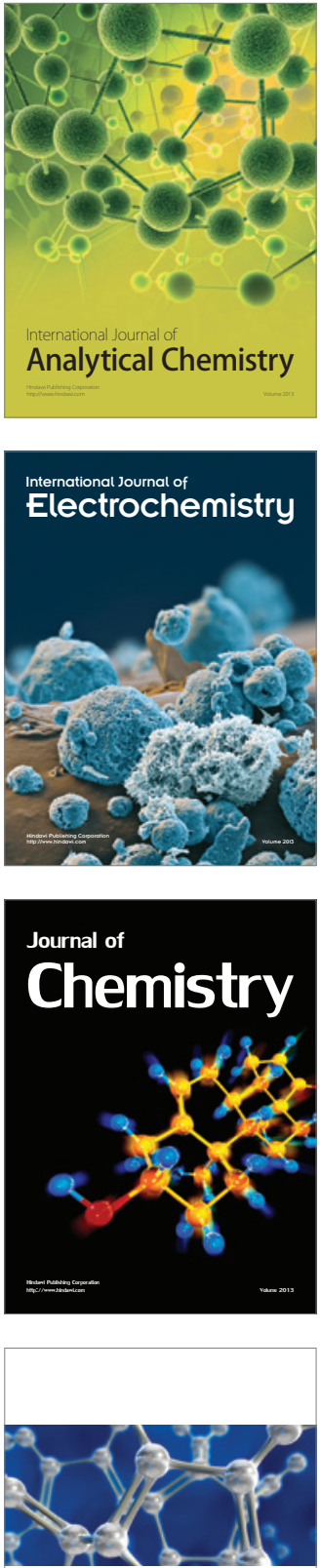

ISRN

Inorganic Chemistry

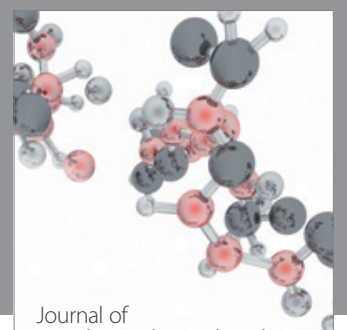

Analytical Methods in Chemistry

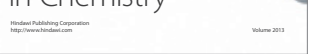

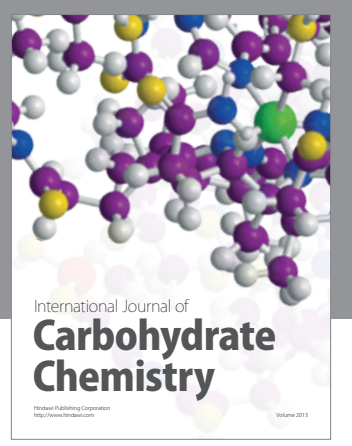
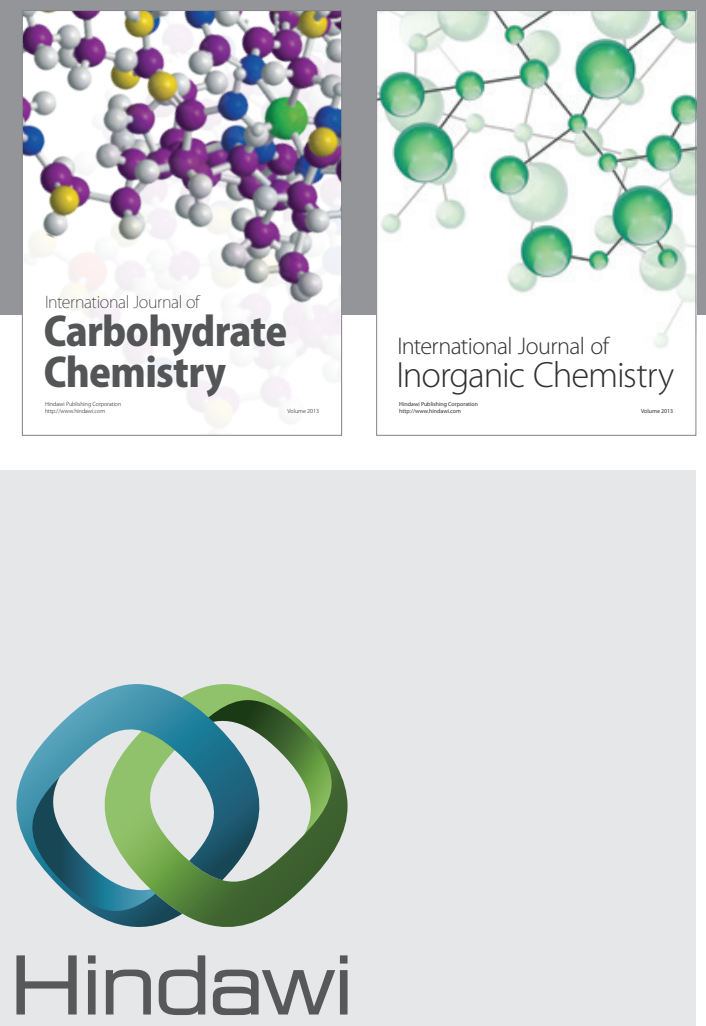

Submit your manuscripts at http://www.hindawi.com
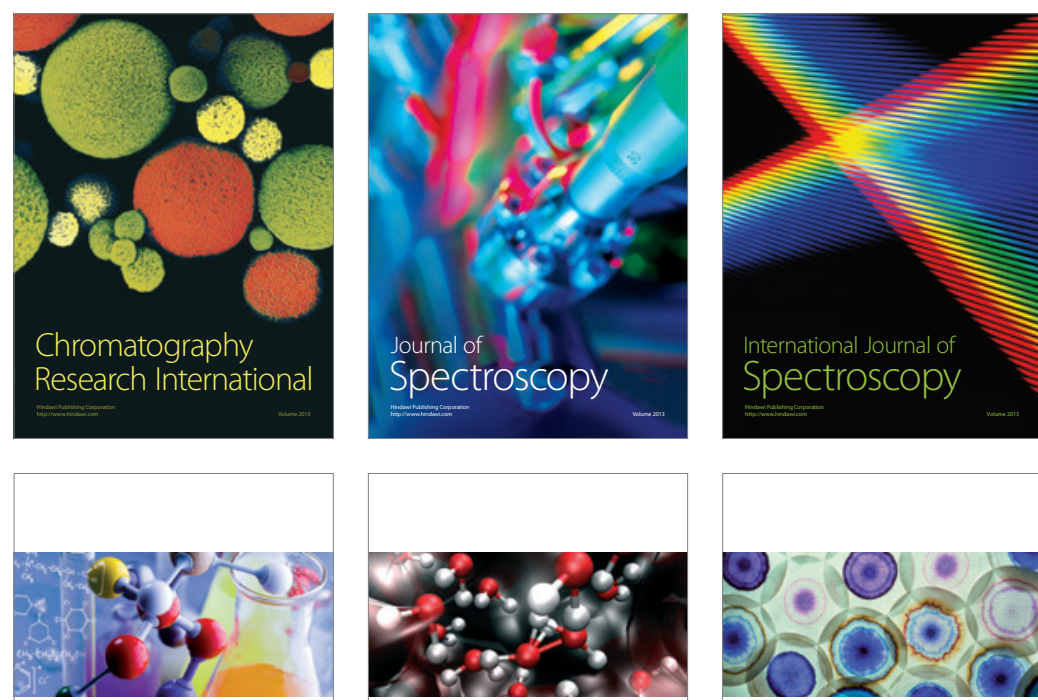

ISRN

ISRN

Organic Chemistry

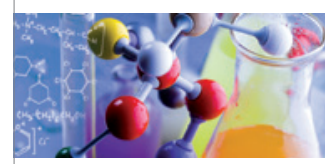

Physical Chemistry

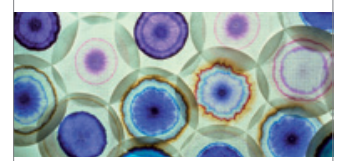

ISRN

Chromatography

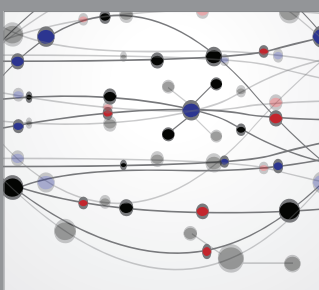

The Scientific World Journal
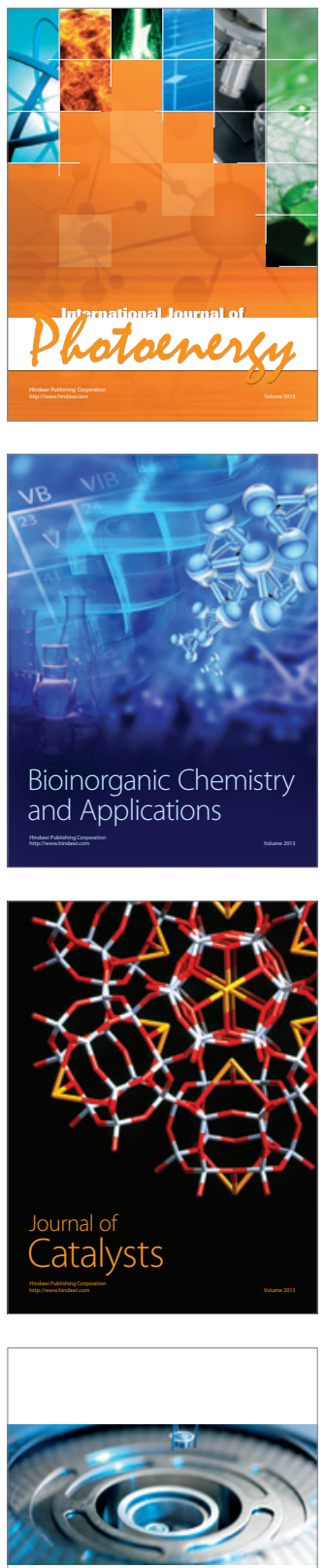

ISRN

Analytical

Chemistry 


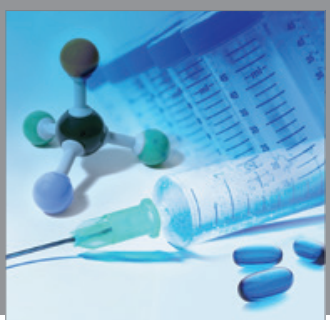

International Journal of

Medicinal Chemistry

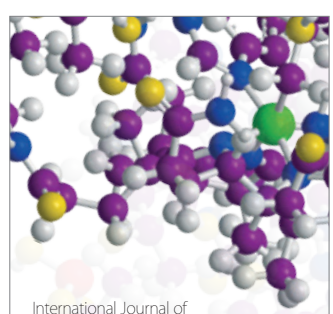

Carbohydrate Chemistry

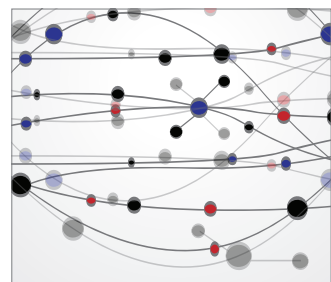

The Scientific World Journal
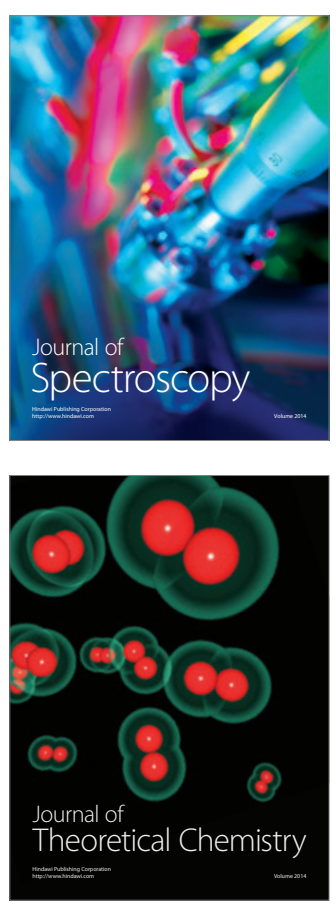
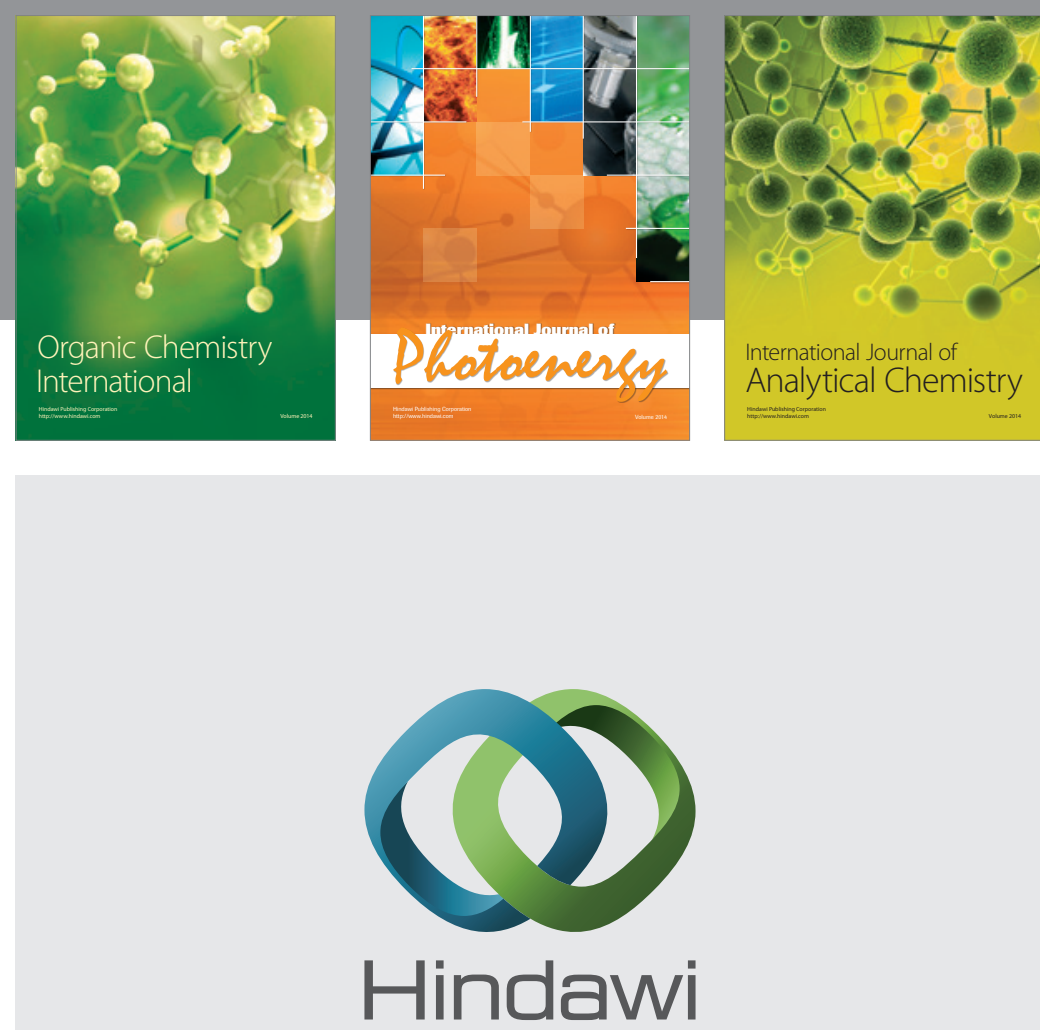

Submit your manuscripts at

http://www.hindawi.com
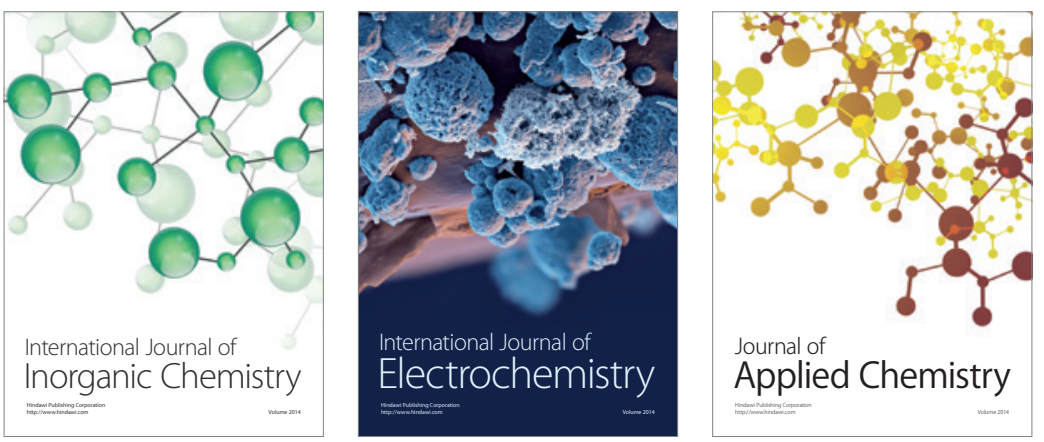

Journal of

Applied Chemistry
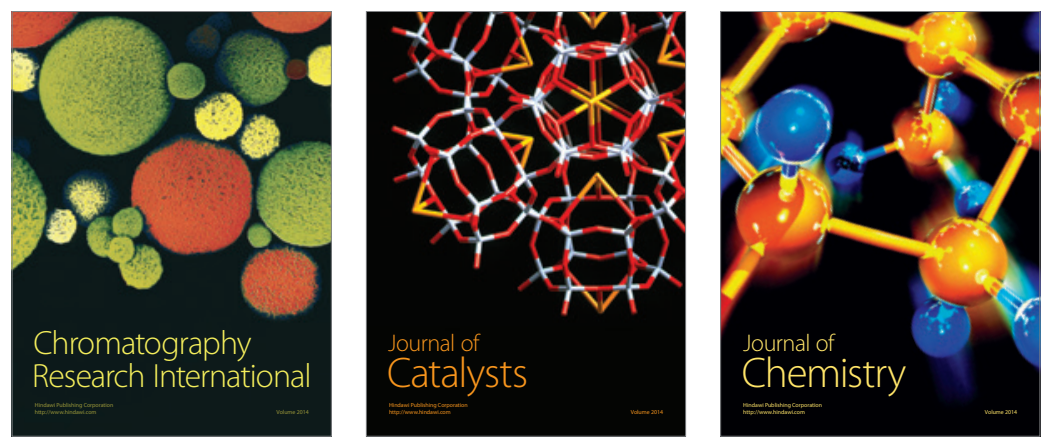
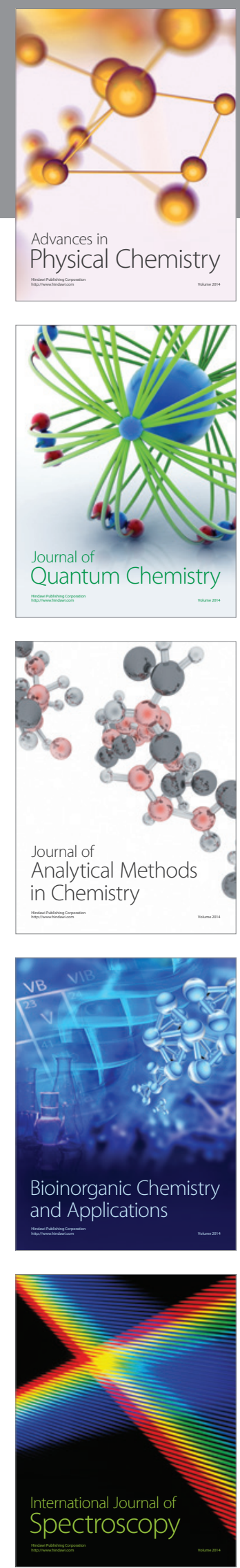